\title{
On new method of estimation of parameters of Size-biased Generalized Gamma Distribution and its Structural properties
}

\author{
A.Ahmed ${ }^{1}$, K.A. $\mathrm{Mir}^{2}$, J. A. Reshi ${ }^{1}$ \\ ${ }^{2}$ Department of Statistics, Govt. Degree College Bemina, Srinagar Kashmir, India. \\ ${ }^{I}$ Department of Statistics, University of Kashmir, India.
}

\begin{abstract}
In this paper, a new class of size-biased Generalized Gamma distribution is introduced. The estimates of parameters of size-biased Generalized Gamma distribution are obtained by using new method of moments. A new distribution which contains as a special case is introduced. The characterizing properties of the model are derived. Also, other important property including Shannon entropy which is the measure of the uncertainty in this class of the distribution are derived and studied.

Key words: Generalized Gamma distribution, Size-biased Generalized Gamma distribution, moment generating function, sample coefficient of variation, Shannon's entropy
\end{abstract}

\section{Introduction}

The Generalized gamma distribution presents a flexible family in the varieties of shapes and hazard functions for modelling duration. It was introduced by Stacy [1]. Distributions that are used in duration analysis in economics include exponential [2,3], lognormal [4], gamma [5], and Weibull [6]. The generalized gamma distribution, which encompasses exponential, gamma, and Weibull as subfamilies, and lognormal as a limiting distribution, has been used in economics by Jasggia [7]. In information theory, the important properties including Shannon entropy and Fisher's information matrix which are the measure of the uncertainty in this class of the distribution are derived and studied. The concept of Shannon entropy [8] is the central role of information theory, some-times referred as measure of uncertainty. The entropy of a random variable is defined in terms of its probability distribution and can be shown to be a good measure of randomness or uncertainty.

Although Prentice [9] have presented a procedure to obtain the three parameters of the generalized gamma distribution, his procedure still quit complicated. In this paper, we propose a simple procedure to obtained three estimators by using its characterization and moment estimation approach. Note that Hwang .T and Huang. P [10] have obtained more general characterizations with the independence of sample coefficient of variation $V_{n}$ with sample mean $\bar{X}_{n}$ as one of its special cases when random samples are drawn from the generalized gamma distribution. Their characterization is used to derive the expectation and the variance of $V_{n}{ }^{2}$ and then the new estimators for the three parameters of size-biased generalized gamma distribution are proposed.

The pdf of the generalized gamma distribution is given by:

$$
f(x ; \lambda, \beta, k)=\frac{\lambda \beta}{\Gamma k}(\lambda x)^{k \beta-1} e^{-(\lambda x)^{\beta}} \text {, for } x>0 \text { and } \lambda, \beta, k>0
$$

The mth non-central moments of GGD is given by

$$
E\left(X^{m}\right)=\frac{\Gamma\left(k+\frac{m}{\beta}\right)}{\lambda^{m} \Gamma k} \quad m=1,2, \ldots \ldots .
$$

\section{Size biased Generalized Gamma Distribution}

A size- biased Generalized Gamma distribution (SBGGMD) is obtained by applying the weights $x^{c}$, where $\mathrm{c}=1$ to the Generalized Gamma distribution.

$$
f_{S}(x ; \lambda, \beta, k)=\int_{0}^{\infty} \frac{x f(x ; \lambda, \beta, k)}{E(X)} d x
$$




$$
\begin{aligned}
& f_{S}(x ; \lambda, \beta, k)=\int_{0}^{\infty} \frac{x \frac{\lambda \beta}{\Gamma k}(\lambda x)^{k \beta-1} e^{-(\lambda x)^{\beta}}}{\frac{\Gamma\left(k+\frac{1}{\beta}\right)}{\lambda \Gamma(k)}} d x \\
& f_{S}(x ; \lambda, \beta, k)=\int_{0}^{\infty} \frac{\lambda \beta}{\Gamma\left(k+\frac{1}{\beta}\right)}(\lambda x)^{k \beta} e^{-(\lambda x)^{\beta}} d x
\end{aligned}
$$

Where $g_{w}(x ; \lambda, \beta, k)$ represents a probability density function. This gives the size -biased generalized gamma distribution (SBGGMD) and its pdf is given by

$$
f_{S}(x ; \lambda, \beta, k)=\frac{\lambda \beta}{\Gamma\left(k+\frac{1}{\beta}\right)}(\lambda x)^{k \beta} e^{-(\lambda x)^{\beta}}
$$

For $\lambda>0, k \geq 0, \beta>0$ and $k \beta>1$

\subsection{Special cases:}

1. When $\beta=k=1$, then Size biased Generalized Gamma distribution reduced to Size-biased exponential distribution and its probability distribution is given by

$$
f_{S}(x ; \lambda)=\lambda^{2} x e^{-\lambda x} \quad, \lambda>0
$$

2. When $\beta=1$ then Size biased Generalized Gamma distribution reduced to size-biased gamma distribution and its probability distribution is given by

$$
f_{S}(x ; \lambda, k+1)=\frac{\lambda^{k+1}}{\Gamma k+1} e^{-\lambda x} x^{k} \quad, \lambda>0, k \geq 0,0<x<\infty
$$

3. When $k=0$ and $\beta=1$ then Size biased Generalized Gamma distribution reduced to exponential distribution and its probability distribution is given by

$$
f_{S}(x ; \lambda)=\lambda e^{-\lambda x} \quad, \lambda>0,0<x<\infty
$$

Theorem 2.2. Let $X=\left(x_{1}, x_{2}, x_{3} \ldots x_{n}\right)$ be an identical independently distributed sample from size-biased generalized gamma distribution

$$
\begin{aligned}
& f_{S}(x ; \lambda, \beta, k)=\frac{\lambda \beta}{\Gamma\left(k+\frac{1}{\beta}\right)}(\lambda x)^{k \beta} e^{-(\lambda x)^{\beta}} \\
& \text { then, } E\left(X^{m}\right)=\frac{\beta \Gamma\left(k+\frac{m+1}{\beta}\right)}{\lambda^{m} \Gamma\left(k+\frac{1}{\beta}\right)} \quad m=1,2, \ldots \ldots . . .
\end{aligned}
$$

Proof: Using equation (2), the mth non central moment is given by,

$$
\begin{aligned}
& E\left(X^{m}\right)=\int_{0}^{\infty} x^{m} \frac{\lambda \beta}{\Gamma\left(k+\frac{1}{\beta}\right)}(\lambda x)^{k \beta} e^{-(\lambda x)^{\beta}} d x \\
& E\left(X^{m}\right)=\frac{\beta \lambda^{k \beta+1}}{\Gamma\left(k+\frac{1}{\beta}\right)} \int_{0}^{\infty} x^{(k \beta+r+1)-1} e^{-(\lambda x)^{\beta}} d x
\end{aligned}
$$


On solving the above equation, we get

$$
E\left(X^{m}\right)=\frac{\beta \Gamma\left(k+\frac{m+1}{\beta}\right)}{\lambda^{m} \Gamma\left(k+\frac{1}{\beta}\right)}
$$

III. New Moment Estimator Of The Size-Biased Generalized Gamma Distribution

For deriving new moment estimators of three parameters of the size-biased generalized gamma distribution, we need the following theorem obtained by using the similar approach of Hwang .T and Huang .P (Theorems of 2006).

Theorem 3.1. Let $n \geq 3$ and let $X_{1}, X_{2}, X_{3} \ldots X_{n}$ be a n positive identical independently distributed random variables having a probability density function $f(x)$. Then the independence of the sample mean $\bar{X}_{n}$ and the sample coefficient of variation $V_{n}=\frac{S_{n}}{\bar{X}_{n}}$ is equivalent to that $f(x)$ is a size-biased generalized gamma density where $S_{n}$ is the sample standard deviation.

The next theorem is easy to prove and need to derive the expectation and the variance of $V_{n}^{2}=\left(\frac{S_{n}}{\bar{X}_{n}}\right)^{2}$, where $\bar{X}_{n}$ and $S_{n}$ are respectively the sample mean and the sample standard deviation.

Theorem 3.2: Let $n \geq 3$ and let $X_{1}, X_{2}, X_{3} \ldots X_{n}$ be a n positive identical independently distributed random samples drawn from a population having a size-biased generalized gamma density

$$
f_{S}(x ; \lambda, \beta, k)=\frac{\lambda \beta}{\Gamma\left(k+\frac{1}{\beta}\right)}(\lambda x)^{k \beta} e^{-(\lambda x)^{\beta}}, x>0, \lambda>0, \beta \geq 0, k>0
$$

Then $E\left(S_{n}{ }^{2}\right)=\frac{\beta\left[\Gamma\left(k+\frac{1}{\beta}\right) \Gamma\left(k+\frac{3}{\beta}\right)-\beta \Gamma\left(k+\frac{2}{\beta}\right)\right]}{\lambda^{2} \Gamma^{2}\left(k+\frac{1}{\beta}\right)}$

Proof :

$$
\begin{aligned}
& \text { Here, } E\left(X^{m}\right)=\frac{\beta \Gamma\left(k+\frac{m+1}{\beta}\right)}{\lambda^{m} \Gamma\left(k+\frac{1}{\beta}\right)}, m=1,2,3, \ldots \\
& E\left(\bar{X}_{n}\right)=\frac{\beta \Gamma\left(k+\frac{2}{\beta}\right)}{\lambda \Gamma\left(k+\frac{1}{\beta}\right)} \\
& E\left(\bar{X}_{n}^{2}\right)=\frac{\beta \Gamma\left(k+\frac{3}{\beta}\right) \Gamma\left(K+\frac{1}{\beta}\right)+(n-1) \beta^{2} \Gamma^{2}\left(k+\frac{2}{\beta}\right)}{n k \Gamma^{2}\left(k+\frac{1}{\beta}\right)}
\end{aligned}
$$

And $E\left(S_{n}^{2}\right)=n \cdot V\left(\bar{X}_{n}\right)$ 


$$
E\left(S_{n}^{2}\right)=\frac{\beta\left[\Gamma\left(k+\frac{1}{\beta}\right) \Gamma\left(k+\frac{3}{\beta}\right)-\beta \Gamma\left(k+\frac{2}{\beta}\right)\right]}{\lambda^{2} \Gamma^{2}\left(k+\frac{1}{\beta}\right)}
$$

Where $\bar{X}_{n}$ and $S_{n}{ }^{2}$ are respectively their sample mean and sample variance.

Theorem 3.3: Let $n \geq 3$ and let $X_{1}, X_{2}, X_{3} \ldots X_{n}$ be a n positive identical independently distributed random samples drawn from a population having a size-biased generalized gamma density

$$
\begin{array}{r}
f_{S}(x ; \lambda, \beta, k)=\frac{\lambda \beta}{\Gamma\left(k+\frac{1}{\beta}\right)}(\lambda x)^{k \beta} e^{-(\lambda x)^{\beta}}, x>0, \lambda>0, \beta \geq 0, k>0 \\
E\left(\frac{S_{n}^{2}}{\bar{X}_{n}}\right)=\frac{n\left[\Gamma\left(k+\frac{3}{\beta}\right) \Gamma\left(k+\frac{1}{\beta}\right)-\beta \Gamma^{2}\left(k+\frac{2}{\beta}\right)\right]}{\Gamma\left(k+\frac{3}{\beta}\right) \Gamma\left(k+\frac{1}{\beta}\right)+(n-1) \beta \Gamma^{2}\left(k+\frac{2}{\beta}\right)}
\end{array}
$$

Then

Where $\bar{X}_{n}$ and $S_{n}{ }^{2}$ are respectively their sample mean and sample variance.

Proof: By theorem 3.1, we have

$$
E\left(S_{n}{ }^{2}\right)=E\left(\frac{S_{n}{ }^{2}}{\bar{X}_{n}{ }^{2}} \cdot \bar{X}_{n}^{2}\right)=E\left(\frac{S_{n}^{2}}{\bar{X}_{n}^{2}}\right) . . E\left(\bar{X}_{n}^{2}\right)
$$

And hence

$$
E\left(\frac{S_{n}^{2}}{\bar{X}_{n}^{2}}\right)=\frac{E\left(S_{n}^{2}\right)}{E\left(\bar{X}_{n}^{2}\right)}
$$

Applying theorem 3.2 to the above identity yields that

$$
E\left(\frac{S_{n}^{2}}{\bar{X}_{n}}\right)=\frac{n\left[\Gamma\left(k+\frac{3}{\beta}\right) \Gamma\left(k+\frac{1}{\beta}\right)-\beta \Gamma^{2}\left(k+\frac{2}{\beta}\right)\right]}{\Gamma\left(k+\frac{3}{\beta}\right) \Gamma\left(k+\frac{1}{\beta}\right)+(n-1) \beta \Gamma^{2}\left(k+\frac{2}{\beta}\right)}
$$

Thus 3.3 is established.

Note that

$$
E\left(\frac{S_{n}^{2}}{\bar{X}_{n}}\right) \rightarrow \frac{\left[\Gamma\left(k+\frac{3}{\beta}\right) \Gamma\left(k+\frac{1}{\beta}\right)\right]}{\beta \Gamma^{2}\left(k+\frac{2}{\beta}\right)}-1 \text { as } n \rightarrow \infty \text { and that this limit is the square of }
$$

the population coefficient of variation. Thus, $\frac{S_{n}{ }^{2}}{\bar{X}_{n}{ }^{2}}$ is an asymptotically unbiased estimator of the square of the population coefficient of variation.

Theorem 3.4: Let $n \geq 3$ and let $X_{1}, X_{2}, X_{3} \ldots X_{n}$ be a n positive identical independently distributed random samples drawn from a population having a size-biased generalized gamma density 
$f_{S}(x ; \lambda, \beta, k)=\frac{\lambda \beta}{\Gamma\left(k+\frac{1}{\beta}\right)}(\lambda x)^{k \beta} e^{-(\lambda x)^{\beta}}, x>0, \lambda>0, \beta>0, k \geq 0$

Then $\quad E\left(e^{t x^{\beta}}\right)=\frac{\lambda^{k \beta+1}}{\left(\lambda^{\beta}-t\right)^{k+\frac{1}{\beta}}}$

Proof: Here $E\left(e^{t x^{\beta}}\right)=\int_{0}^{\infty} e^{t x^{\beta}} f(x ; \lambda, \beta, k) d x$

$$
\begin{aligned}
& E\left(e^{t x^{\beta}}\right)=\int_{0}^{\infty} e^{t x^{\beta}} \frac{\beta \lambda^{k \beta+1}}{\Gamma\left(k+\frac{1}{\beta}\right)} x^{k \beta} e^{-(\lambda x)^{\beta}} d x \\
& E\left(e^{t x^{\beta}}\right)=\frac{\beta \lambda^{k \beta+1}}{\Gamma\left(k+\frac{1}{\beta}\right)} \int_{0}^{\infty} x^{k \beta} e^{-x^{\beta}\left(\lambda^{\beta}-t\right)} d x \\
& \text { Put } x^{\beta}=t \Rightarrow x=t^{\frac{1}{\beta}} \quad d x=\frac{d t}{\beta x^{\beta-1}} \\
& x \rightarrow 0, t \rightarrow 0 \text { and } x \rightarrow \infty, t \rightarrow \infty \\
& E\left(e^{t x^{\beta}}\right)=\frac{\lambda^{k \beta+1}}{\Gamma\left(k+\frac{1}{\beta}\right)} \int_{0}^{\infty} t^{k} e^{-t\left(\lambda^{\beta}-t\right)} \frac{d t}{t^{1-\frac{1}{\beta}}} \\
& E\left(e^{t x^{\beta}}\right)=\frac{\lambda^{k \beta+1}}{\Gamma\left(k+\frac{1}{\beta}\right)} \int_{0}^{\infty} t^{k+\frac{1}{\beta}-1} e^{-t\left(\lambda^{\beta}-t\right)} d t \\
& E\left(e^{t x^{\beta}}\right)=\frac{\lambda^{k \beta+1}}{\Gamma\left(k+\frac{1}{\beta}\right)} \frac{\Gamma\left(k+\frac{1}{\beta}\right)}{\left(\lambda^{\beta}-t\right)^{k+\frac{1}{\beta}}} \\
& E\left(e^{t x^{\beta}}\right)=\frac{\lambda^{k \beta+1}}{\left(\lambda^{\beta}-t\right)^{k+\frac{1}{\beta}}}
\end{aligned}
$$

Substitute $\beta=1$ in the above relation, we have

$$
E\left(e^{t x}\right)=\frac{\lambda^{k+1}}{(\lambda-t)^{k+1}}
$$

\section{Shannon's entropy of size-biased Generalized Gamma Distribution}

For deriving be entropy of the size-biased Generalized Gamma distribution, we need the following two definitions that more details of them can be found in [11].

Definition 3.2.1: The entropy of the discrete alphabet random variable $f$ defined on the probability space is defined by: 


$$
H_{P}(f)=-\sum_{i=1}^{n} p(f=a) \log (p(f=a))
$$

It is obvious that

$$
H_{P}(f) \geq 0
$$

Definition 3.2.1: The oblivious generalizations of the definition of entropy for a probability density function $f$ defined on the real line as:

$$
H(f)=-\int_{0}^{\infty} f(x) \log f(x)=E(-\log (x))
$$

Provided the integral exists.

Theorem 3.5: Let $X_{1}, X_{2}, X_{3} \ldots X_{n}$ be a n positive identical independently distributed random samples drawn from a population having a size-biased generalized gamma density

$$
f_{S}(x ; \lambda, \beta, k)=\frac{\lambda \beta}{\Gamma\left(k+\frac{1}{\beta}\right)}(\lambda x)^{k \beta} e^{-(\lambda x)^{\beta}}, x>0, \lambda>0, \beta>0, k \geq 0
$$

Then Shannon's entropy of Size-biased Generalized Gamma Distribution is:

$$
H(f(x ; \lambda, k, \beta))=-\log \left(\frac{\beta \lambda^{k \beta+1}}{\Gamma\left(k+\frac{1}{\beta}\right)}\right)-k\left[\Psi\left(K+\frac{1}{\beta}\right)-\log \lambda^{\beta}\right]+\lambda^{\beta} \beta \frac{\Gamma\left(k+\frac{2}{\beta}\right)}{\lambda \Gamma\left(k+\frac{1}{\beta}\right)}
$$

Proof: Shannon's entropy is defined as:

$$
\begin{gathered}
H[f(x ; \alpha, \beta, k)]=E[-\log \{f(x ; \alpha, \beta, k\}] \\
H[f(x ; \alpha, \beta, k)]=E\left[-\log \left\{\frac{\beta \lambda^{k \beta+1}}{\Gamma\left(k+\frac{1}{\beta}\right)} x^{k \beta} e^{-(\lambda x)^{\beta}}\right\}\right] \\
H[f(x ; \alpha, \beta, k)]=\left[-\log \left\{\frac{\beta \lambda^{k \beta+1}}{\Gamma\left(k+\frac{1}{\beta}\right)}\right\}-k \beta E(\log x)+\lambda^{\beta} \beta E(x)\right] \\
H[f(x ; \alpha, \beta, k)]=\left[-\log \left\{\frac{\beta \lambda^{k \beta+1}}{\Gamma\left(k+\frac{1}{\beta}\right)}\right\}-k \beta E(\log x)+\lambda^{\beta} \beta E(x)\right] \\
H(f(x ; \lambda, k, \beta))=-\log \left(\frac{\beta \lambda^{k \beta+1}}{\Gamma\left(k+\frac{1}{\beta}\right)}\right)-k \beta E(\log x)+\lambda^{\beta} \beta \frac{\Gamma\left(k+\frac{2}{\beta}\right)}{\lambda \Gamma\left(k+\frac{1}{\beta}\right)}
\end{gathered}
$$

Now, $E(\log (x))=\int_{0}^{\infty} \log x f(x ; \alpha, \beta, k) d x$

$$
E(\log (x))=\int_{0}^{\infty} \log x \frac{\beta \lambda^{k \beta+1}}{\Gamma\left(k+\frac{1}{\beta}\right)} x^{k \beta} e^{-(\lambda x)^{\beta}} d x
$$


Let $(\lambda x)^{\beta}=t \Rightarrow x^{\beta}=\frac{t}{\lambda^{\beta}} \Rightarrow x=\left(\frac{t}{\lambda^{\beta}}\right)^{\frac{1}{\beta}} \quad d x=\frac{d t}{\lambda \beta t^{1-\frac{1}{\beta}}}$

$E(\log (x))=\frac{\beta \lambda^{k \beta+1}}{\Gamma\left(k+\frac{1}{\beta}\right)} \int_{0}^{\infty} \log \left(\frac{t}{\lambda^{\beta}}\right)^{\frac{1}{\beta}}\left(\frac{t}{\lambda^{\beta}}\right)^{k \beta} e^{-t} \frac{d t}{\lambda \beta t^{1-\frac{1}{\beta}}}$

$E(\log (x))=\frac{1}{\Gamma\left(k+\frac{1}{\beta}\right)} \int_{0}^{\infty} \log \left(\frac{t}{\lambda^{\beta}}\right)^{\frac{1}{\beta}} t^{k+\frac{1}{\beta}-1} e^{-t} d t$

$E(\log (x))=\frac{1}{\beta \Gamma\left(k+\frac{1}{\beta}\right)} \int_{0}^{\infty}\left(\log t e^{-t} t^{k+\frac{1}{\beta}-1} d t\right)-\frac{\log \lambda^{\beta}}{\Gamma\left(k+\frac{1}{\beta}\right)} \int_{0}^{\infty} e^{-t} t^{k+\frac{1}{\beta}-1} d t$

$E(\log (x))=\frac{\Gamma^{\prime}\left(k+\frac{1}{\beta}\right)}{\beta \Gamma\left(k+\frac{1}{\beta}\right)}-\frac{\log \lambda^{\beta} \Gamma\left(k+\frac{1}{\beta}\right)}{\beta \Gamma\left(k+\frac{1}{\beta}\right)}$

$E(\log (x))=\frac{\Psi\left(k+\frac{1}{\beta}\right)-\log \lambda^{\beta}}{\beta}$

Substitute the value of equation (14) in equation (12), we have

$H(f(x ; \lambda, k, \beta))=-\log \left(\frac{\beta \lambda^{k \beta+1}}{\Gamma\left(k+\frac{1}{\beta}\right)}\right)-k\left[\Psi\left(K+\frac{1}{\beta}\right)-\log \lambda^{\beta}\right]+\lambda^{\beta} \beta \frac{\Gamma\left(k+\frac{2}{\beta}\right)}{\lambda \Gamma\left(k+\frac{1}{\beta}\right)}$

The relation (15) is the Shannon's entropy of Size-biased Generalized Gamma Distribution.

\section{References}

[1]. Stacy,E.W., A Generalization of the Gamma Distribution, The Annals of Mathematical Statistics, Vol. 33, No.3,pp.1187$1192,(1962) S$

[2]. Diebold F. X., Rudebusch G.D. (1990). "A Nonparametric investigation of duration dependence in the American business cycle,” Journal of Political Economy, 98, 596-616.

[3]. $\quad$ Kiefer N.M. (1984). "Simple test for heterogeneity in exponential models of duration," Journal of Labour Economics, 539-549.

[4]. Eckstein Z., Wolpin K.I (1995). "Duration to first job and the return to schooling estimates from a search-matching model," Review of Economic Studies, 6, 6-6.

[5]. Lancaster T.(1979) “Econometric methods for the duration of unemployment," Econometrica, , 939-956.

[6] . Favero C. A., Pesaran M.H., Sharma S. (1994) "A duration model of irreversible oil investment: theory and empirical evidence," Journal of Applied Econometrics, 9, 95-112.

[7]. Jaggia S. (1991) "Specification tests based on the heterogeneous generalized gamma model of duration: with an application to Kennan's strike data, "Journal of Applied Econometrics, 6, 169-180.

[8] . Jamas Shannon C.E (1948) “A Mathematical theory of communication,” Bell system Technical Journal, 27, 623-659

[9] . Prentice, R.L., A Log gamma model and its maximum likelihood estimation. Biometrika, 61(1974), 539-544.

[10]. Huang, P. H., Hwang, T.Y., On new moment estimation of parameters of the generalized gamma distribution using it's characterization, Taiwanese Journal of Mathematics, Volume 10, No. 4, pp. 1083-1093, (2006).

[11] Cover T. M., Thomas J.A., Elements of Information Theory, New York, Wiley, 1991. 